\title{
Referencing the Polarization Orientation to the Emission-Region Magnetic Field
}

\author{
Joanna M. Rankin \\ Physics Department, University of Vermont, Burlington, VT 05405 USA: \\ Joanna.Rankin@uvm.edu
}

\begin{abstract}
Using the proper-motion as a proxy for a pulsar's rotation-axis orientation, the emission polarization can be related to the magnetic field direction in the emission region, providing a much more physical foundation for understanding orthogonal mode radiation.
\end{abstract}

Keywords. pulsars: general, techniques: polarimetric, radiation mechanisms: nonthermal

For decades we have referred to the orthogonal polarization modes (OPMs) and even used the terms "primary" and "secondary" polarization mode without any specific knowledge of their relationship to the magnetic field orientation in the emission region. Behind this usage was the strong assumption that the "primary" mode must have its electric vector parallel to the projected magnetic field direction - an assumption that we now know from the Vela X-ray arcs and what has followed (Helfand et al. 2001, Johnston et al. 2005; Rankin 2007) to be wrong, wrong, wrong.

Remarkably, the rotation-axis orientation $\boldsymbol{\Omega}$ of many younger pulsars is $\|$ to their proper-motion directions (Rankin 2015), and this provides a means of relating the observed polarization position angles (PPAs) to the magnetic fields in the emission regions. The logic of the analysis demonstrating this circumstance is shown in Figure 1: The top left panel shows that the emitting field lines are $\|$ to $\Omega$ at the "fiducial" instant when the pulsar emission beam faces the Earth. The proper motion can then be used as a proxy for $\boldsymbol{\Omega}$, and the polarization orientation with respect to the "fiducial" field direction established. The top right profile shows a sample of a pulsar that is highly depolarized by OPM mixing; however, the latest trailing-edge PPAs seem to be least distorted and thus reliable. The Venn diagram (lower left) shows how the population of pulsars with core components, absolute polarimetry and accurate proper motions are selected. And finally the lower right distribution shows that most pulsars with core emission exhibit PPAs that are orthogonal to the local magnetic field direction in their emission regions.

Pulsar emission is then comprised of radiation both $\|$ and $\perp$ to the local emission-region magnetic field $\mathbf{B}$. These orthogonally polarized electromagnetic waves then represent the ordinary $(\mathrm{O})$ and extraordinary $(\mathrm{X})$ physical modes. That the latest, deepest core emission is preferentially $\mathrm{X}$ mode indicates strongly that this radiation stems from processes within a dense plasma where the $\mathrm{O}$ mode is strongly absorbed. Such absolute polarimetry offers exciting new possibilities to study pulsar radiation in terms of the physical modes and explore how the $\mathrm{O}$ mode escapes and the circular polarization is generated.

\section{References}

Helfand, D. J., Gotthelf, E. V., \& Halpern, J. P. 2001, ApJ 556, 380

Johnston, S., Hobbs, G., Vigeland, S., Kramer, M., Weisberg, J. M., \& Lyne, A. G. 2005, MNRAS 364, 1397

Rankin, J. M. 2015, ApJ , 804, 112 

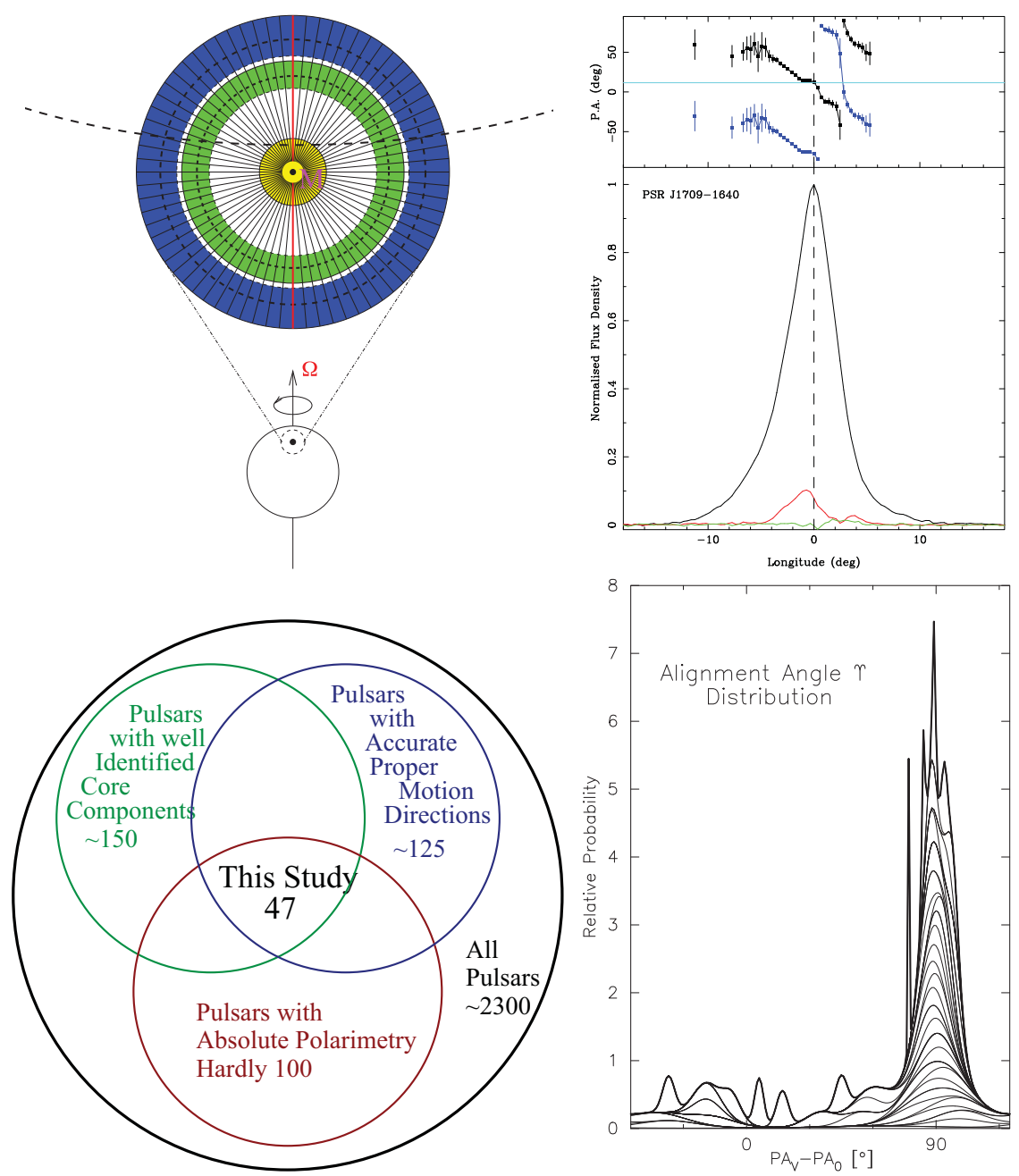

Figure 1. Diagrams showing the logic of our analysis: (Upper Left) Diagram showing the projected field lines associated with pulsar emission at the "fiducial" instant when the beam faces the Earth directly. Both the magnetic axis $\mathbf{M}$ and pulsar rotation axis $\boldsymbol{\Omega}$ are indicated in relation to the "fiducial" field lines (magneta in the on-line figure) associated with the radiation we receive at that instant. The projected fiducial field is $\|$ to the rotation axis $\boldsymbol{\Omega}$ and thus used as a proxy for $\boldsymbol{\Omega}$ which is otherwise usually unobservable. Two conal beams and the central core beam (outer: blue, inner: green, and core: yellow in the on-line figure) are shown in relation to a typical sightline traverse (black dashed). (Upper Right) Polarization profile of B1706-16 from Johnston et al. (2005) showing its highly depolarized single core-component profile at $1369 \mathrm{MHz}$. The roughly linear, negative-going PPA (black curve) plotted in the upper panel connects smoothly with the PPA $-90^{\circ}$ (blue in the on-line figure) curve on the trailing edge of the profile, coinciding with the minimum in the linear polarization (red curve in the on-line figure) in the lower panel where the total power (black) and circular polarization (green in the on-line figure) are also plotted. The PPAs are referred to infinite frequency, and the proper-motion orientation is shown as a horizontal (cyan in the on-line figure) line. (Lower Left) Venn diagram showing the population of pulsars having accurate determinations of three different types. (Lower Right) Distribution showing the core-emission alignment angles $\Psi$ of the 47 pulsars core-emitting pulsars. Here the alignment data is shown as the sum of Gaussians having half widths corresponding to their error values. See Rankin (2015) for details. 